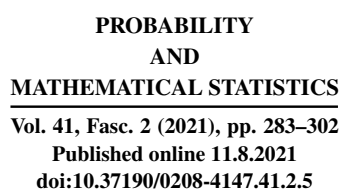

\title{
WICK CALCULUS FOR VECTOR-VALUED GAUSSIAN WHITE NOISE FUNCTIONALS*
}

\author{
BY \\ UN CIG Ji (CheongJu) AND PENG Cheng MA (CheongJu)
}

\begin{abstract}
Based on a Gel'fand triple $(\mathcal{N}) \otimes \mathcal{E} \subset \Gamma(H) \otimes \mathfrak{h} \subset((\mathcal{N}) \otimes \mathcal{E})^{*}$, we introduce a new notion of Wick type product of generalized Gaussian white noise functionals which is associated with a continuous bilinear mapping $\mathfrak{B}: \mathcal{E}^{*} \times \mathcal{E}^{*} \rightarrow \mathcal{E}^{*}$. Then we study Wick type differential equations for vector-valued generalized Gaussian white noise functionals and, as a simple application, we study Wick type differential equations for matrixvalued generalized Gaussian white noise functionals. For our purposes, we make a systematic study of equicontinuity of the left and right Wick type multiplication operators.
\end{abstract}

2020 Mathematics Subject Classification: Primary 60H40; Secondary 46F $25,46 \mathrm{~A} 11$.

Key words and phrases: white noise theory, Gaussian white noise functional, Wick product, equicontinuous, Wick type differential equation.

\section{INTRODUCTION}

Since white noise theory [12] was initiated by Hida [11] to give a rigorous mathematical meaning of white noise as the time derivative of Brownian motion, the theory has been extensively developed as an infinite-dimensional distribution theory [27] with wide applications to classical [3], [14], [28] and quantum stochastic calculus [6], [15], [16], [22], [20], [30], [31], [33], [37], mathematical finance [1], and quantum field theory [21], [22], [23] (see also references therein).

In white noise approach to stochastic and quantum stochastic calculus and quantum field theory, the Wick product and its calculus play an important role and have been studied by many authors [1], [3], [9], [8], [10], [14], [39], [36]. Also, in [4], the $q$-analogs of the Wick product were introduced, and the $q$-Wick product has been studied by several authors (see [25], [7], [17] and references therein).

* Research supported by Basic Science Research Program through the NRF funded by the MEST (NRF-2016R1D1A1B01008782). 
In [19]-[23] the notion of quantum white noise derivatives was introduced and studied; these are defined as commutators with annihilation and creation operators, and so are derivations under the usual operator composition. However, quantum white noise derivatives are also derivations under the Wick product of white noise operators whose derivation properties are useful in several research fields [20], [21], [23]. Also, the annihilation operators satisfy the derivation property under the Wick product of Gaussian white noise functionals [5]. However, the studies of Wick calculus have been mostly concentrated on the case of scalar-valued white noise functionals.

On the other hand, in [31], Obata studied operator calculus on the space of vector-valued Gaussian white noise functionals.

The main purpose of this paper is to study Wick type calculus on vector-valued Gaussian white noise functionals. However, to our knowledge, there is no suitable definition of Wick product of vector-valued white noise distributions on Gaussian space. Hence, we first introduce a new and natural notion of Wick product of vector-valued Gaussian white noise functionals. Our study is based on a Gel'fand triple

$$
(\mathcal{N}) \otimes \mathcal{E} \subset \Gamma(H) \otimes \mathfrak{h} \subset((\mathcal{N}) \otimes \mathcal{E})^{*} \cong(\mathcal{N})^{*} \otimes \mathcal{E}^{*}
$$

The Wick product of scalar-valued Gaussian white noise functionals in $(\mathcal{N})^{*}$ is defined and very well-studied (see Section 22. But, in general, $\mathcal{E}$ is a separable Hilbert (locally convex) space, and so there is no product operation on $\mathcal{E}$. However, if there is a natural product structure on $\mathcal{E}^{*}$ or any (natural) continuous bilinear mapping $\mathfrak{B}: \mathcal{E}^{*} \times \mathcal{E}^{*} \rightarrow \mathcal{E}^{*}$, then to define our new Wick type product, we take the Wick product on the $(\mathcal{N})^{*}$ part and the continuous bilinear mapping $\mathfrak{B}$ on the $\mathcal{E}^{*}$ part. Then we study the Wick type differential equations (see (6.1) and 6.7p) for vector-valued generalized Gaussian white noise functionals. As a simple application, we consider the case of $\mathcal{E}=\mathcal{E}^{*}=M_{d \times d}(\mathbb{C})$, the space of $d \times d$ matrices with complex entries. Then the Wick type differential equations 6.1) and 6.7) become differential equations for matrix-valued generalized Gaussian white noise functionals.

For our purposes, we make a systematic study of equicontinuity of the left and right Wick type multiplication operators; their equicontinuity is characterized in terms of the equicontinuity of $S$-transforms.

This paper is organized as follows. In Sections 2 and 3, we recall some basic notions for scalar and vector-valued Gaussian white noise functionals, respectively. In Section 4, we introduce a new notion of Wick type product of vectorvalued Gaussian white noise functionals, which is associated with a continuous bilinear mapping $\mathfrak{B}: \mathcal{E}^{*} \times \mathcal{E}^{*} \rightarrow \mathcal{E}^{*}$. In Section 5 , we conduct a systematic study of equicontinuity of the left and right Wick type multiplication operators. In Section 6, we study Wick type differential equations, and as an example, we discuss a Wick type differential equation for matrix-valued Gaussian white noise functionals. 


\section{GAUSSIAN WHITE NOISE FUNCTIONALS}

We first recall the standard construction (see [13], [26], [27], [32]) of a Gel'fand triple in white noise theory [11], [12]. We start with the Gel'fand triple $\mathcal{N} \subset$ $H \subset \mathcal{N}^{*}$ constructed from $(H, A)$, where $H$ is a separable complex Hilbert space with inner product $\left\langle\cdot,{ }^{-}\right\rangle$and norm $|\cdot|_{0}$ and $A$ is a densely defined, positive, selfadjoint operator in $H$. We suppose that $\inf \operatorname{Spec}(A)>0$.

For each $p \geqslant 0$, put $\mathcal{N}_{p}=\operatorname{Dom}\left(A^{p}\right)$. Then the dense subspace $\mathcal{N}_{p} \subset H$ becomes a Hilbert space with norm $|\cdot|_{p}$ defined by $|\cdot|_{q}:=\left|A^{q} \cdot\right|_{0}$ (for $\left.q \in \mathbb{R}\right)$. Let $\mathcal{N}_{-p}$ be the completion of $H$ with respect to the norm $|\cdot|_{-p}$. Then by identifying $H$ and $H^{*}$, we have a chain of Hilbert spaces with natural continuous inclusions

$$
\cdots \subset \mathcal{N}_{p} \subset \mathcal{N}_{0}=H \subset \mathcal{N}_{-p} \subset \cdots,
$$

where $\mathcal{N}_{-p}(p \geqslant 0)$ is identified with the strong dual space $\mathcal{N}_{p}^{*}$ of $\mathcal{N}_{p}$ with respect to $H$. Taking the limit spaces

$$
\mathcal{N}:=\underset{p \rightarrow \infty}{\operatorname{proj} \lim } \mathcal{N}_{p}=\bigcap_{p \geqslant 0} \mathcal{N}_{p}, \quad \mathcal{N}^{*} \cong \underset{p \rightarrow \infty}{\operatorname{ind} \lim } \mathcal{N}_{-p}=\bigcup_{p \geqslant 0} \mathcal{N}_{-p},
$$

where $\mathcal{N}^{*}$ is the dual space (equipped with the strong dual topology) of $\mathcal{N}$, we have a triple $\mathcal{N} \subset H \subset \mathcal{N}^{*}$. It is well-known that the separable Hilbert space $\mathcal{N}$ constructed from $(H, A)$ is nuclear if and only if $A^{-r}$ is of Hilbert-Schmidt type for some $r>0$ (see [32, Proposition 1.3.4]). In what follows, we always assume that $A$ is a densely defined, positive self-adjoint operator in $H$ satisfying the following assumptions:

(A1) $\left\|A^{-1}\right\|_{\text {op }}=(\inf \operatorname{Spec}(A))^{-1}<1$,

(A2) $A^{-1}$ is of Hilbert-Schmidt type.

In this case, we obtain a Gel'fand triple $\mathcal{N} \subset H \subset \mathcal{N}^{*}$.

The (boson) Fock space over the Hilbert space $\mathcal{N}_{p}$ is defined by

$$
\Gamma\left(\mathcal{N}_{p}\right)=\left\{\phi=\left(f_{n}\right)_{n=0}^{\infty}: f_{n} \in \mathcal{N}_{p}^{\widehat{\otimes} n},\|\phi\|_{p}^{2}=\sum_{n=0}^{\infty} n !\left|f_{n}\right|_{p}^{2}<\infty\right\}
$$

where $\mathcal{N}_{p}^{\widehat{\otimes} n}$ is the $n$-fold symmetric tensor power of the Hilbert space $\mathcal{N}_{p}$, and $\mathcal{N}_{p}^{\widehat{\otimes} 0}$ is understood as the scalar field $\mathbb{C}$.

Let $\Gamma(A)$ be the second quantization operator of $A$, i.e., $\Gamma(A) \phi=\left(A^{\otimes n} f_{n}\right)_{n=0}^{\infty}$ for $\phi=\left(f_{n}\right)_{n=0}^{\infty} \in \Gamma(H)$. Then the norm $\|\cdot\|_{p}$ in $\Gamma(H)$ is introduced by means of $\Gamma(A)$, namely,

$$
\|\phi\|_{p}^{2}=\sum_{n=0}^{\infty} n !\left|\left(A^{\otimes n}\right)^{p} f_{n}\right|_{0}^{2}=\left\|\Gamma(A)^{p} \phi\right\|_{0}^{2},
$$


and $\Gamma(A)$ becomes a densely defined, positive self-adjoint operator in $\Gamma(H)$. Hence the pair $(\Gamma(H), \Gamma(A))$ yields a chain of Fock spaces with natural continuous inclusions

$$
\cdots \subset \Gamma\left(\mathcal{N}_{p}\right) \subset \cdots \subset \Gamma(H) \subset \cdots \subset \Gamma\left(\mathcal{N}_{-p}\right) \subset \cdots,
$$

where $\Gamma(H)$ and its dual space are identified. Then we have the Gel'fand triple

$$
\underset{p \rightarrow \infty}{\operatorname{proj} \lim } \Gamma\left(\mathcal{N}_{p}\right)=:(\mathcal{N}) \subset \Gamma(H) \subset(\mathcal{N})^{*} \cong \underset{p \rightarrow \infty}{\operatorname{ind} \lim } \Gamma\left(\mathcal{N}_{-p}\right) .
$$

Here $(\mathcal{N})$ and $(\mathcal{N})^{*}$ are mutually dual spaces. We note that $(\mathcal{N})$ is a nuclear space since $A^{-1}$ is of Hilbert-Schmidt type (see (A2)).

Note that the complex Hilbert space $H$ can be represented as the complexification $H=H_{\mathbb{R}}+i H_{\mathbb{R}}$ of a real Hilbert space $H_{\mathbb{R}}$. Then $\mathcal{N}$ and $\mathcal{N}^{*}$ are also represented as the complexifications of real locally convex spaces $\mathcal{N}_{\mathbb{R}}$ and $\mathcal{N}_{\mathbb{R}}^{*}$, respectively. Then by the Bochner-Minlos theorem there exists a unique probability measure $\mu$, called the standard Gaussian measure, on $\mathcal{N}_{\mathbb{R}}^{*}$ such that

$$
\exp \left(-\frac{1}{2}|\xi|_{0}^{2}\right)=\int_{\mathcal{N}_{\mathbb{R}}^{*}} e^{i\langle x, \xi\rangle} \mu(d x), \quad \xi \in \mathcal{N}_{\mathbb{R}},
$$

where $\langle\cdot, \cdot\rangle$ is the canonical $\mathbb{C}$-bilinear form on $\mathcal{N}^{*} \times \mathcal{N}$. The probability space $\left(\mathcal{N}_{\mathbb{R}}^{*}, \mu\right)$ is called the standard Gaussian space. Then the celebrated Wiener-ItôSegal isomorphism is a unitary isomorphism between the boson Fock space $\Gamma(H)$ and the complex Hilbert space $\left(L^{2}\right):=L^{2}\left(\mathcal{N}_{\mathbb{R}}^{*}, \mu\right)$ of complex-valued square integrable Gaussian random variables on $\left(\mathcal{N}_{\mathbb{R}}^{*}, \mu\right)$; the isomorphism is uniquely determined by the correspondence

$$
\left(L^{2}\right) \ni \phi_{\xi}(x)=e^{\langle x, \xi\rangle-\frac{1}{2}\langle\xi, \xi\rangle} \leftrightarrow \phi_{\xi}=\left(\xi^{\otimes n} / n !\right)_{n=0}^{\infty} \in \Gamma(H)
$$

for $\xi \in H$, where $\phi_{\xi}$ is called the exponential vector associated with $\xi \in H$. The Gel'fand triple $(\mathcal{N}) \subset\left(L^{2}\right) \subset(\mathcal{N})^{*}$ of (generalized) Gaussian random variables corresponding to the Gel'fand triple (2.2) is called the Hida-Kubo-Takenaka space [26].

We note that the set of all exponential vectors $\phi_{\xi}$ for $\xi \in \mathcal{N}$ spans a dense subspace of $(\mathcal{N})$. Hence, every white noise distribution in $(\mathcal{N})^{*}$ is uniquely determined by its $S$-transform defined by

$$
F:=S \Phi: \mathcal{N} \ni \xi \mapsto S \Phi(\xi)=\left\langle\left\langle\Phi, \phi_{\xi}\right\rangle\right\rangle \in \mathbb{C}
$$

(see [2], [27], [32], [38]), where $\langle\langle\cdot, \cdot\rangle\rangle$ is the canonical bilinear form on $(\mathcal{N})^{*} \times(\mathcal{N})$. By standard arguments, $F$ satisfies the following conditions:

(C1) for any $\xi, \eta \in \mathcal{N}$, the map $\mathbb{C} \ni z \mapsto F(z \xi+\eta) \in \mathbb{C}$ is entire, 
(C2) there exist constants $C, p \geqslant 0$ such that

$$
|F(\xi)| \leqslant C \exp \left(|\xi|_{p}^{2}\right), \quad \xi \in \mathcal{N}
$$

The following analytic characterization theorem for $S$-transforms of scalarvalued Gaussian white noise functionals was proved by Potthoff and Streit [38], and then extended by many authors with wide applications. For more details, we refer to [2] and references therein.

THEOREM 2.1 ([38]). A function $F: \mathcal{N} \rightarrow \mathbb{C}$ satisfies conditions $(\mathbf{C 1})$ and (C2) if and only if there exists a unique $\Phi \in(\mathcal{N})^{*}$ such that $F=S \Phi$.

Let $\Phi, \Psi \in(\mathcal{N})^{*}$. Then by Theorem 2.1 there exists a unique element, denoted by $\Phi \diamond \Psi$, in $(\mathcal{N})^{*}$ such that

$$
S(\Phi \diamond \Psi)(\xi)=S(\Phi)(\xi) S(\Psi)(\xi), \quad \xi \in \mathcal{N} .
$$

The element $\Phi \diamond \Psi$ is called the Wick product of $\Phi$ and $\Psi$ (see [1], [5], [6], [14], [27], [29], [39]).

\section{VECTOR-VALUED GAUSSIAN WHITE NOISE FUNCTIONALS}

For details of constructing the spaces of vector-valued Gaussian white noise functionals, we refer to [31], for which [32, Proposition 1.3.8] (see also [31, Theorem A.9]) plays an essential role.

Let $\mathfrak{h}$ be another complex Hilbert space whose norm is denoted by $|\cdot|_{0}$ again. This is sometimes called an initial space. Let $B$ be a densely defined, positive self-adjoint operator in $\mathfrak{h}$ satisfying the conditions

(B1) $\inf \operatorname{Spec}(B)>0$,

(B2) $B^{-1}$ is of Hilbert-Schmidt type.

Then by the standard construction, from $(\mathfrak{h}, B)$ we construct a Gel'fand triple

$$
\mathcal{E} \subset \mathfrak{h} \subset \mathcal{E}^{*}
$$

and then from condition (B2), $\mathcal{E}$ is a nuclear space.

Under the above notations and assumptions, $\Gamma(A) \otimes B$ is a densely defined, positive self-adjoint operator in $\Gamma(H) \otimes \mathfrak{h}$ with inf $\operatorname{Spec}(\Gamma(A) \otimes B)>0$.

Then the pair $(\Gamma(H) \otimes \mathfrak{h}, \Gamma(A) \otimes B)$ admits a Gel'fand triple

$$
(\mathcal{N}) \otimes \mathcal{E} \subset \Gamma(H) \otimes \mathfrak{h} \subset((\mathcal{N}) \otimes \mathcal{E})^{*} \cong(\mathcal{N})^{*} \otimes \mathcal{E}^{*}
$$

(see [32, Proposition 1.3.8] and also [31, Theorem A.9]), where

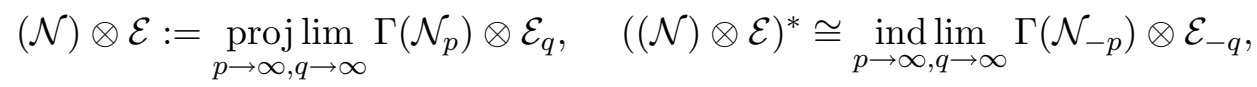


and the topological isomorphism in 3.1) comes from the kernel theorem (see [32, Theorem 1.3.10]). Then $(\mathcal{N}) \otimes \mathcal{E}$ becomes a nuclear space (see [32, Proposition 1.3.7]).

In the above construction, basic notations used so far for scalar-valued functionals are naturally extended to vector-valued ones. The canonical bilinear form on $(\mathcal{N})^{*} \otimes \mathcal{E}^{*} \times(\mathcal{N}) \otimes \mathcal{E}$ is denoted by $\langle\langle\cdot, \cdot\rangle\rangle$ again. Similarly, the norms of $(\mathcal{N}) \otimes \mathcal{E}$ are denoted again by $\|\cdot\|_{p}$, i.e.,

$$
\|\phi\|_{p}^{2}:=\sum_{n=0}^{\infty} n !\left|\left(A^{\otimes n}\right)^{p} \otimes B^{p} F_{n}\right|_{0}^{2}=\left\|\Gamma(A)^{p} \otimes B^{p} \phi\right\|_{0}^{2}
$$

for $\phi=\left(F_{n}\right) \in(\mathcal{N}) \otimes \mathcal{E}$, where $\|\cdot\|_{0}$ is the Hilbertian norm on $\Gamma(H) \otimes \mathfrak{h}$ and $F_{n} \in \mathcal{N}^{\widehat{\otimes} n} \otimes \mathcal{E}$.

We note that $\left\{\phi_{\xi} \otimes u: \xi \in \mathcal{N}, u \in \mathcal{E}\right\}$ spans a dense subspace of $(\mathcal{N}) \otimes \mathcal{E}$, where $\phi_{\xi}$ is an exponential vector defined in (2.3). Similarly to the case of scalarvalued Gaussian white noise functionals, the $S$-transform of $\Phi \in((\mathcal{N}) \otimes \mathcal{E})^{*}$ is defined as an $\mathcal{E}^{*}$-valued function on $\mathcal{N}$ by

$$
\langle S \Phi(\xi), u\rangle=\left\langle\left\langle\Phi, \phi_{\xi} \otimes u\right\rangle\right\rangle, \quad \xi \in \mathcal{N}, u \in \mathcal{E} .
$$

Then we have the following analytic characterization theorem for the $S$-transform of vector-valued Gaussian white noise functionals.

THEOREM 3.1 ([31]). A function $F: \mathcal{N} \rightarrow \mathcal{E}^{*}$ satisfies the conditions

(F1) for any fixed $\xi, \eta \in \mathcal{N}$ and $u \in \mathcal{E}$, the function $z \mapsto\langle F(\xi+z \eta), u\rangle \in \mathbb{C}$ is entire on $\mathbb{C}$,

(F2) there exist $C, K, p \geqslant 0$ such that

$$
|\langle F(\xi), u\rangle| \leqslant C|u|_{p} \exp \left(K|\xi|_{p}^{2}\right), \quad \xi \in \mathcal{N}, u \in \mathcal{E},
$$

if and only if there exists $a \Phi \in((\mathcal{N}) \otimes \mathcal{E})^{*}$ such that $S \Phi=F$.

The following result is an analytic characterization of the convergence of Gaussian white noise functionals in terms of their $S$-transforms, which is useful for various applications. The result for scalar-valued Gaussian white noise functionals can be found in [27].

THEOREM 3.2. Let $\Phi_{n} \in(\mathcal{N})^{*} \otimes \mathcal{E}^{*} \cong((\mathcal{N}) \otimes \mathcal{E})^{*}$ and $U_{n}=S \Phi_{n}$. Then $\Phi_{n}$ converges strongly in $(\mathcal{N})^{*} \otimes \mathcal{E}^{*}$ if and only if the following conditions are satisfied:

(N1) $\lim _{n \rightarrow \infty}\left\langle U_{n}(\xi), u\right\rangle$ exists for all $\xi \in \mathcal{N}$ and $u \in \mathcal{E}$,

(N2) there exist constants $C>0, K>0, p \geqslant 0$ such that

$$
\left|\left\langle U_{n}(\xi), u\right\rangle\right| \leqslant C|u|_{p} \exp \left(K|\xi|_{p}^{2}\right), \quad \forall n \in \mathbb{N}, \xi \in \mathcal{N}, u \in \mathcal{E} .
$$

Proof. The proof is a modification of the proof for the scalar-valued case (see [27] and also [35], [24]). 


\section{WICK PRODUCT}

Let $\mathfrak{L}: \mathcal{E}^{*} \otimes \mathcal{E}^{*} \rightarrow \mathcal{E}^{*}$ be a continuous linear map. Then by the construction of $\mathcal{E}^{*} \otimes \mathcal{E}^{*}$ (see [31, Theorem A.9] and [32, Theorem 1.3.10]), we can see that for any $p \geqslant 0$ there exists $q \geqslant 0$ such that $\mathfrak{L}$ is continuous from $\mathcal{N}_{-p} \otimes \mathcal{N}_{-p}$ into $\mathcal{N}_{-(p+q)}$, and so there exists $C \geqslant 0$ such that

$$
|\mathfrak{L}(x \otimes y)|_{-(p+q)} \leqslant C|x|_{-p}|y|_{-p}, \quad x, y \in \mathcal{N}_{-p} .
$$

For the continuous canonical bilinear map $\chi: \mathcal{E}^{*} \times \mathcal{E}^{*} \rightarrow \mathcal{E}^{*} \otimes \mathcal{E}^{*}$ defined by $\chi(x, y)=x \otimes y$, the continuous bilinear map $\mathfrak{L} \circ \chi$ is denoted by $\mathfrak{B}:=\mathfrak{B}_{\mathfrak{L}}$, i.e., for any $x, y \in \mathcal{E}^{*}$,

$$
\mathfrak{B}(x, y)=\mathfrak{B}_{\mathfrak{L}}(x, y)=\mathfrak{L}(x \otimes y) .
$$

The following theorem is useful to introduce the Wick product of vector-valued Gaussian white noise functionals.

THEOREM 4.1. Let $\mathfrak{B}$ be as in (4.1). For $\Phi, \Psi \in((\mathcal{N}) \otimes \mathcal{E})^{*}$, there exists a unique $\Theta \in((\mathcal{N}) \otimes \mathcal{E})^{*}$ such that for all $\xi \in \mathcal{N}$ and $u \in \mathcal{E}$,

$$
\langle S \Theta(\xi), u\rangle=\langle\mathfrak{B}(S \Phi(\xi), S \Psi(\xi)), u\rangle .
$$

Proof. Let $\Phi, \Psi \in((\mathcal{N}) \otimes \mathcal{E})^{*}, \xi \in \mathcal{N}$ and $u \in \mathcal{E}$ be given. Then for the adjoint operator $\mathfrak{L}^{*}: \mathcal{E} \rightarrow \mathcal{E} \otimes \mathcal{E}$ of $\mathfrak{L}$, we have

$$
\langle\mathfrak{B}(S \Phi(\xi), S \Psi(\xi)), u\rangle=\langle\mathfrak{L}(S \Phi(\xi) \otimes S \Psi(\xi)), u\rangle=\left\langle S \Phi(\xi) \otimes S \Psi(\xi), \mathfrak{L}^{*} u\right\rangle .
$$

Since $\mathfrak{L}^{*} u \in \mathcal{E} \otimes \mathcal{E}$, there exists a sequence $\left\{w_{n}\right\}_{n=0}^{\infty} \subset \mathcal{E} \otimes_{\text {alg }} \mathcal{E}$ such that $w_{n} \rightarrow \mathfrak{L}^{*} u$ in $\mathcal{E} \otimes \mathcal{E}$ as $n \rightarrow \infty$. Then

$$
\left\langle S \Phi(\xi) \otimes S \Psi(\xi), \mathfrak{L}^{*} u\right\rangle=\lim _{n \rightarrow \infty}\left\langle S \Phi(\xi) \otimes S \Psi(\xi), w_{n}\right\rangle .
$$

For each given $\eta \in \mathcal{N}$, we denote the functions

$$
\begin{aligned}
& \mathbb{C} \ni z \mapsto\left\langle S \Phi(\xi+z \eta) \otimes S \Psi(\xi+z \eta), \mathfrak{L}^{*} u\right\rangle, \\
& \mathbb{C} \ni z \mapsto\left\langle S \Phi(\xi+z \eta) \otimes S \Psi(\xi+z \eta), w_{n}\right\rangle
\end{aligned}
$$

by $f$ and $f_{n}(n \in \mathbb{N})$, respectively. Then by applying (F2) of Theorem 3.1, we find that for some constants $C_{\Phi}, C_{\Psi}, p \geqslant 0$,

$$
\begin{aligned}
\left|f_{n}(z)\right| & \leqslant C_{\Phi} C_{\Psi}\left|w_{n}\right|_{p} \exp \left(K_{\Phi}|\xi+z \eta|_{p}^{2}\right) \exp \left(K_{\Psi}|\xi+z \eta|_{p}^{2}\right) \\
& \leqslant C \exp \left(K|z|^{2}\right)
\end{aligned}
$$


for some constants $C, K \geqslant 0$. Since $f_{n}(z)(n \in \mathbb{N})$ are entire functions, by the dominated convergence theorem we obtain

$$
\oint_{\gamma} f(z) d z=\oint_{\gamma} \lim _{n \rightarrow \infty} f_{n}(z) d z=\lim _{n \rightarrow \infty} \oint_{\gamma} f_{n}(z) d z=0
$$

for every closed piecewise $C^{1}$ curve $\gamma$ in $\mathbb{C}$, and so by Morera's theorem, $f(z)$ is entire, which means that $\mathfrak{B}(S \Phi(\xi), S \Psi(\xi))$ satisfies condition (F1).

On the other hand, for each $\Upsilon \in((\mathcal{N}) \otimes \mathcal{E})^{*}$, from $(\mathbf{F 2})$ of Theorem 3.1, there exist constants $C, K, p \geqslant 0$ such that for any $\xi \in \mathcal{N}$,

$$
|\langle S \Upsilon(\xi), u\rangle| \leqslant C|u|_{p} \exp \left(K|\xi|_{p}^{2}\right), \quad u \in \mathcal{E},
$$

which implies that

$$
|S \Upsilon(\xi)|_{-p}=\sup _{|u|_{p} \leqslant 1}|\langle S \Upsilon(\xi), u\rangle| \leqslant C \exp \left(K|\xi|_{p}^{2}\right), \quad \xi \in \mathcal{N} .
$$

Therefore, for given $\Phi, \Psi \in((\mathcal{N}) \otimes \mathcal{E})^{*}$, there exist constants $C_{0}, K_{0}, p_{0} \geqslant 0$ (depending on both $\Phi$ and $\Psi$ ) such that

$$
|S \Phi(\xi)|_{-p_{0}} \leqslant C_{0} \exp \left(K_{0}|\xi|_{p_{0}}^{2}\right), \quad|S \Psi(\xi)|_{-p_{0}} \leqslant C_{0} \exp \left(K_{0}|\xi|_{p_{0}}^{2}\right)
$$

for all $\xi \in \mathcal{N}$, and then by the continuity of $\mathfrak{L}$, there exists $q_{0} \geqslant 0$ such that

$$
|\mathfrak{L}(S \Phi(\xi) \otimes S \Psi(\xi))|_{-\left(p_{0}+q_{0}\right)} \leqslant C_{1}|S \Phi(\xi)|_{-p_{0}}|S \Psi(\xi)|_{-p_{0}} .
$$

Therefore, for any $\xi \in \mathcal{N}$ and $u \in \mathcal{E}$, we obtain

$$
\begin{aligned}
|\langle\mathfrak{B}(S \Phi(\xi), S \Psi(\xi)), u\rangle| & =|\langle\mathfrak{L}(S \Phi(\xi) \otimes S \Psi(\xi)), u\rangle| \\
& \leqslant C_{1}|u|_{p_{0}+q_{0}}|S \Phi(\xi)|_{-p_{0}}|S \Psi(\xi)|_{-p_{0}} \\
& \leqslant C_{0}^{2} C_{1}|u|_{p_{0}+q_{0}} \exp \left(2 K_{0}|\xi|_{p_{0}}^{2}\right) \\
& \leqslant C_{0}^{2} C_{1}|u|_{p_{0}+q_{0}} \exp \left(2 K_{0} \|\left. A^{-1}\left|{ }^{q_{0}}\right| \xi\right|_{q_{0}+p_{0}} ^{2}\right),
\end{aligned}
$$

and so by taking $C=C_{0}^{2} C_{1}, K=2 K_{0}\left\|A^{-1}\right\|^{q_{0}}$ and $p=p_{0}+q_{0}$, we see that $\mathfrak{B}(S \Phi(\xi), S \Psi(\xi))$ satisfies condition (F2) of Theorem 3.1. Thus, by Theorem 3.1 . there exists a unique $\Theta \in((\mathcal{N}) \otimes \mathcal{E})^{*}$ such that $(4.2)$ holds.

From now on, we assume that $\mathfrak{L}: \mathcal{E}^{*} \otimes \mathcal{E}^{*} \rightarrow \mathcal{E}^{*}$ is a continuous linear map and $\mathfrak{B}: \mathcal{E}^{*} \times \mathcal{E}^{*} \rightarrow \mathcal{E}^{*}$ is a continuous bilinear map such that $\mathfrak{L}$ and $\mathfrak{B}$ are related as in 4.1. Then by applying Theorem 4.1. the Wick product, with respect to the continuous bilinear mapping $\mathfrak{B}$, of vector-valued Gaussian white noise functionals is well defined as follows.

Definition 4.1. Let $\Phi, \Psi \in((\mathcal{N}) \otimes \mathcal{E})^{*}$ be given. Then by Theorem 4.1, there exists a unique $\Theta \in((\mathcal{N}) \otimes \mathcal{E})^{*}$ such that 4.2 holds. Then the Gaussian white noise functional $\Theta$ is denoted by $\Phi \diamond_{\mathfrak{B}} \Psi$ and called the $\mathfrak{B}$-Wick product. 
Let $\Phi, \Psi \in((\mathcal{N}) \otimes \mathcal{E})^{*}$ be given. Then by the definition of $\mathfrak{B}$-Wick product, it is obvious that for all $\xi \in \mathcal{N}$,

$$
S\left(\Phi \diamond_{\mathfrak{B}} \Psi\right)(\xi)=\mathfrak{B}(S \Phi(\xi), S \Psi(\xi))=\mathfrak{L}(S \Phi(\xi) \otimes S \Psi(\xi)) .
$$

From Definition 4.1 it is obvious that if the bilinear mapping $\mathfrak{B}$ is symmetric, i.e., $\mathfrak{B}(u, v)=\mathfrak{B}(v, u)$ for all $u, v \in \mathcal{E}^{*}$, then the $\mathfrak{B}$-Wick product is commutative, i.e., $\Phi \diamond_{\mathfrak{B}} \Psi=\Psi \diamond_{\mathfrak{B}} \Phi$ for any $\Phi, \Psi \in(\mathcal{N})^{*} \otimes \mathcal{E}^{*}$. In general, $\mathfrak{B}$ is not symmetric and so the $\mathfrak{B}$-Wick product is noncommutative.

EXAMPLE 4.1. Let $v \in \mathcal{E}$ be fixed. Consider the continuous linear map $\mathfrak{L}$ : $\mathcal{E}^{*} \otimes \mathcal{E}^{*} \rightarrow \mathcal{E}^{*}$ and the continuous bilinear map $\mathfrak{B}: \mathcal{E}^{*} \times \mathcal{E}^{*} \rightarrow \mathcal{E}^{*}$ defined by

$$
\mathfrak{L}(x \otimes y)=x(v) y=\langle x, v\rangle y=\mathfrak{B}(x, y), \quad x, y \in \mathcal{E}^{*} .
$$

Indeed, for any $p, q \geqslant 0$,

$$
\begin{aligned}
|\mathfrak{B}(x, y)|_{-(p+q)} & =|\langle x, v\rangle||y|_{-(p+q)} \leqslant|v|_{p+q}|x|_{-(p+q)}|y|_{-(p+q)} \\
& \leqslant|v|_{p+q}\left\|B^{-1}\right\|^{2 q}|x|_{-p}|y|_{-p},
\end{aligned}
$$

which implies the continuity of $\mathfrak{L}$ and $\mathfrak{B}$. Then for any $\Phi, \Psi \in((\mathcal{N}) \otimes \mathcal{E})^{*}$, we can define the $\mathfrak{B}$-Wick product $\Phi \diamond_{\mathfrak{B}} \Psi$. In this case, for any $u \in \mathcal{E}$,

$$
\left.\left\langle S\left(\Phi \diamond_{\mathfrak{B}} \Psi\right)(\xi), u\right\rangle=\langle\mathfrak{B}(S \Phi(\xi), S \Psi(\xi)), u\rangle=\langle S \Phi(\xi), v\rangle\langle S \Psi(\xi)), u\right\rangle .
$$

EXAMPLE 4.2. Let $\left(\mathfrak{h}^{\prime},\langle\cdot \mid \cdot\rangle\right)$ be a complex Hilbert space and let $\left\{e_{j}\right\}_{j \in J}$ be an orthonormal basis of $\mathfrak{h}^{\prime}$. Let $\mathfrak{h}=\mathcal{L}_{2}\left(\mathfrak{h}^{\prime}\right)$ be the space of all bounded linear operators of Hilbert-Schmidt type on $\mathfrak{h}^{\prime}$. Then $\left(\mathfrak{h},|\cdot|_{0}\right)$ is a Hilbert space with inner product

$$
\langle U \mid V\rangle_{2}:=\sum_{j \in J}\left\langle U e_{j} \mid V e_{j}\right\rangle=\sum_{j \in J}\left\langle V^{*} U e_{j} \mid e_{j}\right\rangle=\operatorname{Tr}\left(V^{*} U\right)
$$

for all $U, V \in \mathfrak{h}=\mathcal{L}_{2}\left(\mathfrak{h}^{\prime}\right)$, and $|U|_{0}^{2}=\langle U \mid U\rangle_{2}=\operatorname{Tr}\left(U^{*} U\right)$ for $U \in \mathfrak{h}$.

Let $B$ be a densely defined, positive linear operator on $\mathfrak{h}$ such that inf $\operatorname{Spec}(B)$ $>1$ and $B^{-r}$ is of Hilbert-Schmidt type. Then from the pair $(\mathfrak{h}, B)$, we construct a Gel'fand triple $\mathcal{E} \subset \mathfrak{h} \subset \mathcal{E}^{*}$. In particular, if $\mathfrak{h}^{\prime}$ is a $d$-dimensional Hilbert (Euclidean) space, then $\mathfrak{h}=M_{d \times d}(\mathbb{C})$ is the space of all $d \times d$ matrices, and then every invertible operator $B$ on $\mathfrak{h}$ is bounded. Therefore, $\mathcal{E}=\mathfrak{h}=M_{d \times d}(\mathbb{C})=\mathcal{E}^{*}$. As an example of a continuous linear mapping $\mathfrak{B}: \mathcal{E}^{*} \times \mathcal{E}^{*} \rightarrow \mathcal{E}^{*}$, consider matrix multiplication, i.e.,

$$
\mathfrak{B}(U, V)=U V, \quad U, V \in \mathcal{E}^{*}=\mathfrak{h}=M_{d \times d}(\mathbb{C}) .
$$

Then for $\Phi, \Psi \in(\mathcal{N})^{*} \otimes \mathcal{E}^{*}=(\mathcal{N})^{*} \otimes \mathfrak{h}$, the $\mathfrak{B}$-Wick product of $\Phi$ and $\Psi$ is characterized by $S\left(\Phi \diamond_{\mathfrak{B}} \Psi\right)(\xi)=\mathfrak{B}(S \Phi(\xi), S \Psi(\xi))=S \Phi(\xi) S \Psi(\xi)$ for $\xi \in \mathcal{N}$. 
EXAMPLE 4.3. Let $\mathfrak{h}=\Gamma\left(\mathfrak{h}^{\prime}\right)$ be the Fock space over the Hilbert space $\mathfrak{h}^{\prime}$ and let $B=\Gamma\left(B^{\prime}\right)$ be the second quantization of a densely defined, positive self-adjoint operator $B^{\prime}$ on $\mathfrak{h}^{\prime}$ such that inf $\operatorname{Spec}\left(B^{\prime}\right)>0$ and $\left(B^{\prime}\right)^{-1}$ is of Hilbert-Schmidt type. Then from the pair $(\mathfrak{h}, B)$ we construct a Gel'fand triple

$$
(E) \subset \mathfrak{h}=\Gamma\left(\mathfrak{h}^{\prime}\right) \subset(E)^{*},
$$

which is an example of a Gel' fand triple $\mathcal{E} \subset \mathfrak{h} \subset \mathcal{E}^{*}$, i.e., $\mathcal{E}=(E)$ and $\mathcal{E}^{*}=(E)^{*}$, where the Gel'fand triple $E \subset \mathfrak{h}^{\prime} \subset E^{*}$ is constructed from $\left(\mathfrak{h}^{\prime}, B^{\prime}\right)$.

For any $U, V \in \mathcal{E}^{*}=(E)^{*}$, the convolution $U * V$ is defined by

$$
U * V=U \diamond V \diamond g,
$$

where $g \in(E)^{*}$ is uniquely determined by $S g(\xi)=e^{\frac{1}{2}\langle\xi, \xi\rangle}$ for $\xi \in E$. We now consider the following examples of continuous bilinear mappings $\mathfrak{B}_{i}: \mathcal{E}^{*} \times \mathcal{E}^{*} \rightarrow$ $\mathcal{E}^{*}(i=1,2)$ :

$$
\begin{array}{ll}
\mathfrak{B}_{1}(U, V)=U \diamond V & \text { for } U, V \in \mathcal{E}^{*}=(E)^{*}, \\
\mathfrak{B}_{2}(U, V)=U * V & \text { for } U, V \in \mathcal{E}^{*}=(E)^{*} .
\end{array}
$$

Here the continuity of the convolution and Wick product can be checked by applying the following: there exist $p, q \geqslant 0$ such that

$$
\|U * V\|_{-(p+q)}=\|U \diamond V \diamond g\|_{-(p+q)} \leqslant\|g\|_{-p}\|U\|_{-p}\|V\|_{-p}
$$

for $U, V \in(E)^{*}($ see [27]).

Then for $\Phi, \Psi \in(\mathcal{N})^{*} \otimes(E)^{*}$, the $\mathfrak{B}_{i}$-Wick products $\Phi \diamond_{\mathfrak{B}_{i}} \Psi$ are characterized by

$$
\begin{aligned}
& S\left(\Phi \diamond_{\mathfrak{B}_{1}} \Psi\right)(\xi)=S \Phi(\xi) \diamond S \Psi(\xi) \\
& S\left(\Phi \diamond_{\mathfrak{B}_{2}} \Psi\right)(\xi)=S \Phi(\xi) * S \Psi(\xi)
\end{aligned}
$$

for all $\xi \in \mathcal{N}$.

\section{EQUICONTINUITY OF MULTIPLICATION OPERATORS}

In the next section, we will study Wick type differential equations for vectorvalued Gaussian white noise functionals. For our study, the notion of equicontinuity is useful, and so we first review some results in [34], and then characterize the equicontinuity of Wick type multiplication operators in terms of the $S$-transform. Let $\mathcal{L}(\mathcal{X}, \mathcal{Y})$ be the space of all continuous linear operators from a locally convex space $\mathcal{X}$ into another locally convex space $\mathcal{Y}$.

Let $T$ be a densely defined, positive self-adjoint operator on a Hilbert space $\mathfrak{H}$ such that $\inf \operatorname{Spec}(T)>0$ and $T^{-1}$ is of Hilbert-Schmidt type. Let $\mathfrak{X} \subset \mathfrak{H} \subset \mathfrak{X}^{*}$ be the Gel'fand triple constructed from $(\mathfrak{H}, T)$ (see Section 2). 
Let $\operatorname{GL}\left(\mathfrak{X}^{*}\right)$ be the group of all linear homeomorphisms from $\mathfrak{X}^{*}$ onto $\mathfrak{X}^{*}$. A family $\left\{\Omega_{z}\right\}_{z \in \mathbb{C}} \subset \mathrm{GL}\left(\mathfrak{X}^{*}\right)$ is called a one-parameter subgroup if $\Omega_{0}=I$ (the identity operator on $\mathfrak{X}^{*}$ ) and $\Omega_{z_{1}} \Omega_{z_{2}}=\Omega_{z_{1}+z_{2}}$ for any $z_{1}, z_{2} \in \mathbb{C}$. A oneparameter subgroup $\left\{\Omega_{z}\right\}_{z \in \mathbb{C}} \subset \mathrm{GL}\left(\mathfrak{X}^{*}\right)$ is said to be holomorphic if the limit

$$
\lim _{z \rightarrow 0} \frac{\Omega_{z} x-x}{z}
$$

exists in $\mathfrak{X}^{*}$ for each $x \in \mathfrak{X}^{*}$. An operator $\Xi \in \mathcal{L}\left(\mathfrak{X}^{*}, \mathfrak{X}^{*}\right)$ is called the infinitesimal generator of the one-parameter subgroup $\left\{\Omega_{z}\right\}_{z \in \mathbb{C}}$ if

$$
\Xi x=\lim _{z \rightarrow 0} \frac{\Omega_{z} x-x}{z}, \quad x \in \mathfrak{X}^{*} .
$$

The following proposition can be proved by applying [34, Proposition 1.3] and duality.

PROPOSITION 5.1. Let $\left\{\Omega_{z}\right\}_{z \in \mathbb{C}} \subset \mathrm{GL}\left(\mathfrak{X}^{*}\right)$ be a holomorphic one-parameter subgroup with infinitesimal generator $\Xi \in \mathcal{L}\left(\mathfrak{X}^{*}, \mathfrak{X}^{*}\right)$. Then

$$
\left\langle\Omega_{z} x, u\right\rangle=\sum_{n=0}^{\infty} \frac{z^{n}}{n !}\left\langle\Xi^{n} x, u\right\rangle, \quad z \in \mathbb{C}, x \in \mathfrak{X}^{*}, u \in \mathfrak{X},
$$

where the series converges absolutely. In particular, a holomorphic one-parameter subgroup is uniquely determined by the infinitesimal generator.

Lemma 5.1. Let $\Xi \in \mathcal{L}(\mathfrak{X}, \mathfrak{X})$. Then for any $p \geqslant 0$, there exist constants $C, q \geqslant 0$ such that $|\Xi u|_{p} \leqslant C|u|_{q}$ for all $u \in \mathfrak{X}$ if and only if for any $p \geqslant 0$, there exist constants $C, q \geqslant 0$ such that $\left|\Xi^{*} x\right|_{-q} \leqslant C|x|_{-p}$ for all $x \in \mathfrak{X}^{*}$, where $\Xi^{*} \in \mathcal{L}\left(\mathfrak{X}^{*}, \mathfrak{X}^{*}\right)$ is the adjoint operator of $\Xi$ with respect to the canonical bilinear form $\langle\cdot, \cdot\rangle$ on $\mathfrak{X}^{*} \times \mathfrak{X}$.

Proof. The proof is straightforward by using duality.

A family $\left\{\Xi_{i}\right\}_{i \in \mathbb{N}} \subset \mathcal{L}\left(\mathfrak{X}^{*}, \mathfrak{X}^{*}\right)$ of operators is said to be equicontinuous if for any $p \geqslant 0$, there exist $q, K \geqslant 0$ such that

$$
\left|\Xi_{i} x\right|_{-q} \leqslant K|x|_{-p}, \quad x \in \mathfrak{X}^{*}, i \in \mathbb{N},
$$

where $|x|_{-p}$ can be infinity for some $x \in \mathfrak{X}^{*}$.

In [34], Obata studied equicontinuous operators from $\mathfrak{X}$ into itself. By applying Lemma 5.1, the results in [34] are also applicable to equicontinuous operators from $\mathfrak{X}^{*}$ into itself. The following lemma is a simple modification of [34, Lemma 2.1]. 
Lemma 5.2. For $\Xi \in \mathcal{L}\left(\mathfrak{X}^{*}, \mathfrak{X}^{*}\right)$ and $\boldsymbol{\Omega}=\left\{\Omega_{z}\right\}_{z \in \mathbb{C}} \subset \mathrm{GL}\left(\mathfrak{X}^{*}\right)$, the following four conditions are equivalent:

(i) there is $R>0$ such that $\left\{(R \Xi)^{n} / n !: n=0,1,2, \ldots\right\}$ is equicontinuous,

(ii) $\left\{(R \Xi)^{n} / n !: n=0,1,2, \ldots\right\}$ is equicontinuous for any $R>0$,

(iii) $\Xi$ is the infinitesimal generator of a holomorphic one-parameter subgroup $\Omega$ such that $\left\{\Omega_{z}:|z|<R\right\}$ is equicontinuous for some $R>0$,

(iv) $\Xi$ is the infinitesimal generator of a holomorphic one-parameter subgroup $\Omega$ such that $\left\{\Omega_{z}:|z|<R\right\}$ is equicontinuous for any $R>0$.

Proof. The proof is straightforward by applying [34, Lemma 2.1] (see also [31]) and Lemma 5.1.

If the bilinear mapping $\mathfrak{B}: \mathcal{E}^{*} \times \mathcal{E}^{*} \rightarrow \mathcal{E}^{*}$ is continuous, for each given $u \in \mathcal{E}^{*}$ we denote the left and right multiplication operators by $M_{u}^{l}$ and $M_{v}^{r}$, respectively, i.e., $M_{u}^{l} v=\mathfrak{B}(u, v)$ and $M_{u}^{r} v=\mathfrak{B}(v, u)$ for all $v \in \mathcal{E}^{*}$. Then it is obvious that for each $u \in \mathcal{E}^{*}, M_{u}^{l}$ and $M_{u}^{r}$ belong to $\mathcal{L}\left(\mathcal{E}^{*}, \mathcal{E}^{*}\right)$. Similarly, for given $\Phi \in((\mathcal{N}) \otimes \mathcal{E})^{*}$, we denote the left and right $\diamond_{\mathfrak{B}}$-multiplication operators by $\mathbf{M}_{\Phi}^{l}$ and $\mathbf{M}_{\Phi}^{r}$, respectively, i.e., $\mathbf{M}_{\Phi}^{l} \Psi=\Phi \diamond_{\mathfrak{B}} \Psi$ and $\mathbf{M}_{\Phi}^{r} \Psi=\Psi \diamond_{\mathfrak{B}} \Phi$ for all $\Psi \in((\mathcal{N}) \otimes \mathcal{E})^{*}$. It is obvious that for any $\xi \in \mathcal{N}$,

$$
S\left(\mathbf{M}_{\Phi}^{l} \Psi\right)(\xi)=M_{S \Phi(\xi)}^{l} S \Psi(\xi), \quad S\left(\mathbf{M}_{\Phi}^{r} \Psi\right)(\xi)=M_{S \Phi(\xi)}^{r} S \Psi(\xi) .
$$

THEOREM 5.1. Let $\Phi \in((\mathcal{N}) \otimes \mathcal{E})^{*}$. Then $\left\{\left(\mathbf{M}_{\Phi}^{l}\right)^{n} / n !\right\}_{n=0}^{\infty}$ is equicontinuous if and only if for any $\xi \in \mathcal{N}$ and $p \geqslant 0$, there exist constants $C, K, q \geqslant 0$ such that

$$
\left|\frac{\left(M_{S \Phi(\xi)}^{l}\right)^{n}}{n !} w\right|_{-q} \leqslant C e^{K|\xi|_{q}^{2}}|w|_{-p}, \quad w \in \mathcal{E}^{*} .
$$

Proof. Suppose that $\left\{\left(\mathbf{M}_{\Phi}^{l}\right)^{n} / n !\right\}_{n=0}^{\infty}$ is equicontinuous. Then for any $p \geqslant 0$, there exist $q, C \geqslant 0$ such that for any $\Psi \in((\mathcal{N}) \otimes \mathcal{E})^{*}$,

$$
\left\|\left(\left(\mathbf{M}_{\Phi}^{l}\right)^{n} / n !\right) \Psi\right\|_{-q} \leqslant C\|\Psi\|_{-p} .
$$

Therefore, from (5.3), for any $\xi \in \mathcal{N}$ and $w \in \mathcal{E}^{*}$, we obtain

$$
\begin{aligned}
\left|\left(\left(M_{S \Phi(\xi)}^{l}\right)^{n} / n !\right) w\right|_{-q} & \leqslant \sup _{|u|_{q} \leqslant 1}\left|\left\langle\left(\left(M_{S \Phi(\xi)}^{l}\right)^{n} / n !\right) w, u\right\rangle\right| \\
& =\sup _{|u|_{q} \leqslant 1}\left|\left\langle S\left(\left(\left(\mathbf{M}_{\Phi}^{l}\right)^{n} / n !\right)\left(\phi_{0} \otimes w\right)\right)(\xi), u\right\rangle\right| \\
& \leqslant \sup _{|u|_{q} \leqslant 1}\left|\left\langle\left(\left(\mathbf{M}_{\Phi}^{l}\right)^{n} / n !\right)\left(\phi_{0} \otimes w\right), \phi_{\xi} \otimes u\right\rangle\right|,
\end{aligned}
$$


and so we have

$$
\begin{aligned}
\left|\left(\left(M_{S \Phi(\xi)}^{l}\right)^{n} / n !\right) w\right|_{-q} & \leqslant \sup _{|u|_{q} \leqslant 1}\left\|\left(\left(\mathbf{M}_{\Phi}^{l}\right)^{n} / n !\right)\left(\phi_{0} \otimes w\right)\right\|\left\|_{-q}\right\| \phi_{\xi} \otimes u \|_{q} \\
& \leqslant C e^{|\xi|_{q}^{2} / 2}|w|_{-p},
\end{aligned}
$$

which implies that for any $\xi \in \mathcal{N},\left\{\left(M_{S \Phi(\xi)}^{l}\right)^{n} / n !\right\}_{n=0}^{\infty}$ is equicontinuous.

Conversely, suppose that (5.4) holds. Then, for any given $R>0$, by Lemma 5.2 (in fact, by modifying the proof of Lemma 5.2), for any $\xi \in \mathcal{N}$ and $p \geqslant 0$, there exist constants $C, K, q \geqslant 0$ such that

$$
\left|\frac{\left(R M_{S \Phi(\xi)}^{l}\right)^{n}}{n !} w\right|_{-q} \leqslant C e^{K|\xi|_{q}^{2}}|w|_{-p}, \quad w \in \mathcal{E}^{*} .
$$

Therefore, for any $\Psi \in((\mathcal{N}) \otimes \mathcal{E})^{*}$,

$$
\left|\left(\left(R M_{S \Phi(\xi)}^{l}\right)^{n} / n !\right) S \Psi(\xi)\right|_{-q} \leqslant C e^{K|\xi|_{q}^{2}}|S \Psi(\xi)|_{-p} \leqslant C\|\Psi\|_{-p} e^{K|\xi|_{q}^{2}} e^{|\xi|_{p}^{2} / 2}
$$

which implies that for any $u \in \mathcal{E}$,

$$
\begin{aligned}
\left|\left\langle S\left(\left(\left(R \mathbf{M}_{\Phi}^{l}\right)^{n} / n !\right) \Psi\right)(\xi), u\right\rangle\right| & \leqslant C|u|_{q}\|\Psi\|_{-p} e^{(K+1)|\xi|_{p \vee q}^{2}} \\
& \leqslant C|u|_{p \vee q}\|\Psi\|_{-p} e^{(K+1)|\xi|_{p \vee q}^{2}} .
\end{aligned}
$$

Hence by Theorem 3.1 there exists $r>0$ such that for all $\Psi \in((\mathcal{N}) \otimes \mathcal{E})^{*}$,

$$
\left\|\left(\left(R \mathbf{M}_{\Phi}^{l}\right)^{n} / n !\right) \Psi\right\|_{-(p \vee q+r)} \leqslant C\|\Psi\|_{-p},
$$

and hence $\left\{\left(\mathbf{M}_{\Phi}^{l}\right)^{n} / n !\right\}_{n=0}^{\infty}$ is equicontinuous.

Corollary 5.1. Let $\Phi \in((\mathcal{N}) \otimes \mathcal{E})^{*}$. Suppose that for any $\xi \in \mathcal{N}$ and $p \geqslant 0$, there exist constants $C, K, q \geqslant 0$ such that (5.4) holds. Then $\mathbf{M}_{\Phi}^{l}$ is the infinitesimal generator of a holomorphic one-parameter subgroup $\left\{\Omega_{z}\right\}_{z \in \mathbb{C}}$ such that $\left\{\Omega_{z}:|z|<R\right\}$ is equicontinuous for any $R>0$. In particular, for each $z \in \mathbb{C}, \Omega_{z}$ has series expansion

$$
\Omega_{z} \Psi=\sum_{n=0}^{\infty} \frac{z^{n}}{n !}\left(\mathbf{M}_{\Phi}^{l}\right)^{n} \Psi=\sum_{n=1}^{\infty} \frac{z^{n}}{n !} \overbrace{\left(\Phi \diamond_{\mathfrak{B}}\left(\cdots \diamond_{\mathfrak{B}}\left(\Phi \diamond_{\mathfrak{B}} \Psi\right) \cdots\right)\right)}^{n \text { times }}
$$

for all $\Psi \in((\mathcal{N}) \otimes \mathcal{E})^{*}$, which converges in $((\mathcal{N}) \otimes \mathcal{E})^{*}$.

Proof. From Theorem 5.1, we see that $\left\{\left(\mathbf{M}_{\Phi}^{l}\right)^{n} / n !\right\}_{n=0}^{\infty}$ is equicontinuous, and so by Lemma 5.2, $\mathbf{M}_{\Phi}^{l}$ is the infinitesimal generator of a holomorphic oneparameter subgroup $\left\{\Omega_{z}\right\}_{z \in \mathbb{C}}$ such that $\left\{\Omega_{z}:|z|<R\right\}$ is equicontinuous for any $R>0$. Therefore, $\Omega_{z}$ has series expansion 5.5 . 
REMARK 5.1. By similar arguments, we can prove that if for any $\xi \in \mathcal{N}$ and $p \geqslant 0$, there exist constants $C, K, q \geqslant 0$ such that

$$
\left|\frac{\left(M_{S \Phi(\xi)}^{r}\right)^{n}}{n !} x\right|_{-q} \leqslant C e^{K|\xi|_{q}^{2}}|x|_{-p}, \quad x \in \mathcal{E}^{*}
$$

then $\mathbf{M}_{\Phi}^{r}$ is the infinitesimal generator of a holomorphic one-parameter subgroup $\left\{\Omega_{z}\right\}_{z \in \mathbb{C}}$ such that $\left\{\Omega_{z}:|z|<R\right\}$ is equicontinuous for any $R>0$. In particular, for each $z \in \mathbb{C}, \Omega_{z}$ has series expansion

$$
\Omega_{z} \Psi=\sum_{n=0}^{\infty} \frac{z^{n}}{n !}\left(\mathbf{M}_{\Phi}^{r}\right)^{n} \Psi=\sum_{n=1}^{\infty} \frac{z^{n}}{n !}\left(\left(\cdots(\Psi \diamond_{\mathfrak{B}} \overbrace{\left.\left.\Phi) \diamond_{\mathfrak{B}} \cdots\right) \diamond_{\mathfrak{B}} \Phi\right)}^{n \text { times }}\right.\right.
$$

for all $\Psi \in((\mathcal{N}) \otimes \mathcal{E})^{*}$, which converges in $((\mathcal{N}) \otimes \mathcal{E})^{*}$.

There is an interesting example to illustrate the condition (5.4) for equicontinuity of $\diamond_{\mathfrak{B}}$-multiplications.

EXAMPLE 5.1. Under the notations and assumptions of Example 4.2, suppose that $\mathfrak{h}^{\prime}$ is a $d$-dimensional Hilbert (Euclidean) space and the continuous bilinear mapping $\mathfrak{B}$ is matrix multiplication. Let $U$ be a $d \times d$ matrix and denote by $|U|_{0}$ the (Hilbert-Schmidt) norm of $U$. Then for any $\Phi \in(\mathcal{N})^{*} \otimes \mathfrak{h}$ and any $\xi \in \mathcal{N}$, $S \Phi(\xi) \in \mathfrak{h}=M_{d \times d}(\mathbb{C})$, and so for any $V \in \mathfrak{h}$ and $n \in \mathbb{N}$, we obtain

$$
\left|\frac{\left(M_{S \Phi(\xi)}^{l}\right)^{n}}{n !} V\right|_{0}=\left|\frac{(S \Phi(\xi))^{n}}{n !} V\right|_{0} \leqslant \frac{\|S \Phi(\xi)\|_{\mathrm{op}}^{n}}{n !}|V|_{0}
$$

for all $\xi \in \mathcal{N}$ and $n \in \mathbb{N}$, where $\|\cdot\|_{\text {op }}$ is the operator norm. Therefore, if for any $\xi \in \mathcal{N}$ and $p \geqslant 0$, there exist constants $C, K, q \geqslant 0$ such that

$$
\frac{\|S \Phi(\xi)\|_{\text {op }}^{n}}{n !} \leqslant C e^{K|\xi|_{q}^{2}} \quad \text { for all } n \in \mathbb{N},
$$

then by Corollary 5.1, $\mathbf{M}_{\Phi}^{l}$ is the infinitesimal generator of a holomorphic oneparameter subgroup $\left\{\Omega_{z}\right\}_{z \in \mathbb{C}}$. In this case, for each $z \in \mathbb{C}, \Omega_{z}$ has series expansion

$$
\Omega_{z} \Psi=\sum_{n=0}^{\infty} \frac{z^{n}}{n !}\left(\mathbf{M}_{\Phi}^{l}\right)^{n} \Psi=\sum_{n=1}^{\infty} \frac{z^{n}}{n !} \overbrace{\left(\Phi \diamond _ { \mathfrak { B } } \left(\cdots \diamond_{\mathfrak{B}}(\Phi\right.\right.}^{n \text { times }} \diamond_{\mathfrak{B}} \Psi) \cdots))
$$

for all $\Psi \in((\mathcal{N}) \otimes \mathcal{E})^{*}$.

\section{WICK TYPE DIFFERENTIAL EQUATIONS}

In this section, we study Wick type differential equations for vector-valued Gaussian white noise processes. Consider the following $\mathfrak{B}$-Wick type differential equation:

$$
\frac{d \Phi(t)}{d t}=\sigma \diamond_{\mathfrak{B}} \Phi(t)+\Psi(t), \quad t \geqslant 0, \quad \Phi(0)=\Phi_{0} \in(\mathcal{N})^{*} \otimes \mathcal{E}^{*}
$$


where $\sigma \in(\mathcal{N})^{*} \otimes \mathcal{E}^{*}$ is independent of the time parameter $t$, and $\{\Phi(t)\}_{t \geqslant 0}$ and $\{\Psi(t)\}_{t \geqslant 0}$ are vector-valued Gaussian white noise processes in $(\mathcal{N})^{*} \otimes \mathcal{E}^{*}$. From now on, we assume that $t \mapsto \Psi(t) \in(\mathcal{N})^{*} \otimes \mathcal{E}^{*}$ is continuous.

First, we consider the homogeneous case.

THEOREM 6.1. Let $\Phi_{0} \in(\mathcal{N})^{*} \otimes \mathcal{E}^{*}$. Suppose that for any $\xi \in \mathcal{N}$ and $p \geqslant 0$, there exist constants $C, K, q \geqslant 0$ such that

$$
\left|\frac{\left(M_{S \sigma(\xi)}^{l}\right)^{n}}{n !} x\right|_{-q} \leqslant C e^{K|\xi|_{q}^{2}}|x|_{-p}, \quad x \in \mathcal{E}^{*} .
$$

If $\Psi(t)=0$, then equation 6.1 has a unique continuously differentiable solution $\{\Phi(t)\}_{t \in \mathbb{R}} \subset(\mathcal{N})^{*} \otimes \mathcal{E}^{*}$ given by

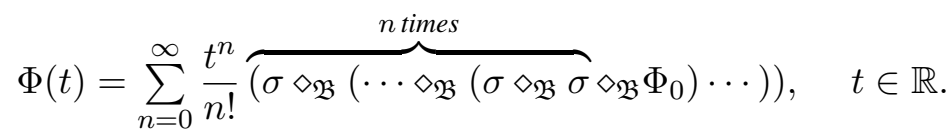

Proof. By Corollary 5.1, $\mathbf{M}_{\sigma}^{l}$ is the infinitesimal generator of a continuously differentiable one-parameter subgroup $\left\{\Omega_{t}\right\}_{t \in \mathbb{R}}$ such that $\left\{\Omega_{t}:|t|<R\right\}$ is equicontinuous for any $R>0$. Hence, $\Phi(t)=\Omega_{t} \Phi_{0}$ is a continuously differentiable solution of (6.1) and by applying (5.5), we have the explicit form of $\Phi(t)$ given in 6.3.

THEOREM 6.2. Let I be a compact interval and let $\{\Psi(t)\}_{t \in I} \subset((\mathcal{N}) \otimes \mathcal{E})^{*}$ be a continuous process. Then there exists $\Upsilon \in((\mathcal{N}) \otimes \mathcal{E})^{*}$, denoted by $\int_{I} \Psi(t) d t$, such that for all $\xi \in \mathcal{N}$ and $u \in \mathcal{E}$,

$$
\langle S \Upsilon(\xi), u\rangle=\int_{I}\langle S(\Psi(t))(\xi), u\rangle d t
$$

Proof. Since $\Psi: I \rightarrow((\mathcal{N}) \otimes \mathcal{E})^{*}$ is continuous, there exists $p \geqslant 0$ such that $\Psi: I \rightarrow\left(\mathcal{N}_{-p}\right) \otimes \mathcal{E}_{-p}$ is continuous (see [35, Proposition A.1]). Therefore, $\left\{\|\Psi(t)\|_{-p}\right\}_{t \in I}$ is bounded, say by $C$, and so for any $\xi \in \mathcal{N}$ and $u \in \mathcal{E}$,

$$
|\langle S(\Psi(t))(\xi), u\rangle|=\left|\left\langle\left\langle\Psi(t), \phi_{\xi} \otimes u\right\rangle\right\rangle\right| \leqslant\|\Psi(t)\|_{-p}|u|_{p} e^{|\xi|_{p}^{2} / 2} \leqslant C|u|_{p} e^{|\xi|_{p}^{2} / 2},
$$

which implies that

$$
\left|\int_{I}\langle S(\Psi(t))(\xi), u\rangle d t\right| \leqslant \int_{I}|\langle S(\Psi(t))(\xi), u\rangle| d t \leqslant m(I) C|u|_{p} e^{|\xi|_{p}^{2} / 2},
$$

where $m$ is the Lebesgue measure. Also, for any closed piecewise $C^{1}$ curve $\gamma$ in $\mathbb{C}$, we obtain

$$
\int_{\gamma} \int_{I}\langle S(\Psi(t))(z \xi+\eta), u\rangle d t d z=\iint_{I}\langle S(\Psi(t))(z \xi+\eta), u\rangle d z d t=0
$$


for any $\xi, \eta \in \mathcal{N}$ and $u \in \mathcal{E}$. Therefore, by applying Theorem 3.1, we see that there exists $\Upsilon \in((\mathcal{N}) \otimes \mathcal{E})^{*}$ such that (6.4) holds.

By applying arguments similar to those used for Theorem 3.2 , we see that

$$
\frac{d}{d t} \int_{0}^{t} \Psi(s) d s=\Psi(t), \quad 0 \leqslant t \leqslant T,
$$

where $\{\Psi(t)\}_{0 \leqslant t \leqslant T} \subset((\mathcal{N}) \otimes \mathcal{E})^{*}(T>0)$ is a continuous process.

Denote by $\overleftarrow{W e x p}_{\mathfrak{B}}(t \sigma) \diamond_{\mathfrak{B}} \Phi_{0}$ the series on the right hand side of 6 6.3), i.e.

$$
\overleftarrow{\operatorname{Wexp}}_{\mathfrak{B}}(t \sigma) \diamond_{\mathfrak{B}} \Phi_{0}=\sum_{n=0}^{\infty} \frac{t^{n}}{n !} \overbrace{\left(\sigma \diamond _ { \mathfrak { B } } \left(\cdots \diamond _ { \mathfrak { B } } \left(\sigma \diamond_{\mathfrak{B}} \sigma\right.\right.\right.}^{n \text { times }} \diamond_{\mathfrak{B}} \Phi_{0}) \cdots)) .
$$

Now, we consider the inhomogeneous case of equation 6.1).

THEOREM 6.3. Let $\Phi_{0} \in(\mathcal{N})^{*} \otimes \mathcal{E}^{*}$. Suppose that for any $\xi \in \mathcal{N}$ and $p \geqslant 0$, there exist constants $C, K, q \geqslant 0$ such that 6.2) holds. Then equation 6.1] has a unique continuously differentiable solution $\{\Phi(t)\}_{t \in \mathbb{R}} \subset(\mathcal{N})^{*} \otimes \mathcal{E}^{*}$ given by

$$
\Phi(t)=\overleftarrow{\operatorname{Wexp}}_{\mathfrak{B}}(t \sigma) \diamond_{\mathfrak{B}} \Phi_{0}+\overleftarrow{\mathrm{Wexp}}_{\mathfrak{B}}(t \sigma) \diamond_{\mathfrak{B}}\left(\int_{0}^{t} \overleftarrow{\operatorname{Wexp}}_{\mathfrak{B}}(-s \sigma) \diamond_{\mathfrak{B}} \Psi(s) d s\right)
$$

for all $t \in \mathbb{R}$.

Proof. By applying Theorem 6.2, we see that the integral in 6.6. is well defined. Then from Theorem 6.1 it is obvious that $\overleftarrow{W e x p}_{\mathfrak{B}}(t \sigma) \diamond_{\mathfrak{B}} \Phi_{0}$ is continuously differentiable, and so, by $(6.5), \Phi(t)$ given by $(6.6)$ is continuously differentiable. For each $\Psi \in(\mathcal{N})^{*} \otimes \mathcal{E}^{*}$ and any $t \in \mathbb{R}$, we put

$$
\Upsilon(t)=\overleftarrow{\mathrm{Wexp}}_{\mathfrak{B}}(t \sigma) \diamond_{\mathfrak{B}}\left(\overleftarrow{\mathrm{Wexp}}_{\mathfrak{B}}(-t \sigma) \diamond_{\mathfrak{B}} \Psi\right)
$$

Then

$$
\frac{d \Upsilon(t)}{d t}=\sigma \diamond_{\mathfrak{B}} \Upsilon(t)-\sigma \diamond_{\mathfrak{B}} \Upsilon(t)=0,
$$

which implies that $\Upsilon(t)$ is constant and $\Upsilon(0)=\Psi$, and hence $\Upsilon(t)=\Psi$, i.e.,

$$
\overleftarrow{\mathrm{Wexp}}_{\mathfrak{B}}(t \sigma) \diamond_{\mathfrak{B}}\left(\overleftarrow{\mathrm{Wexp}}_{\mathfrak{B}}(-t \sigma) \diamond_{\mathfrak{B}} \Psi\right)=\Psi
$$

for all $\Psi \in(\mathcal{N})^{*} \otimes \mathcal{E}^{*}$. Therefore, by direct computation, $\Phi(t)$ is a solution of 6.1].

To prove the uniqueness of solution of 6.1], we suppose that $\Theta(t)$ is a solution of 6.1. Put

$$
F(t)=\Phi(t)-\Theta(t)
$$


Then $\frac{d F(t)}{d t}=\sigma \diamond_{\mathfrak{B}}(F(t))$ and $F(0)=\Phi_{0}-\Phi_{0}=0$. Therefore, from the uniqueness of solution of 6.1 with $\Psi(t)=0$, we have $F(t)=0$, and so $\Theta(t)=\Phi(t)$ for all $t \in \mathbb{R}$. Hence $\Phi(t)$ given by (6.6) is the unique solution of 6.1).

Put

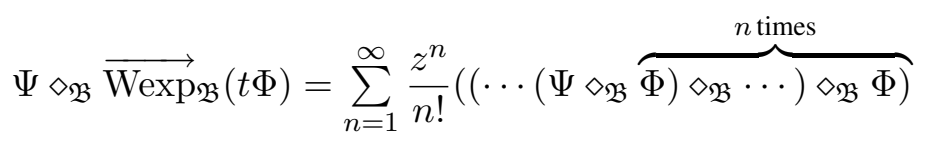

for $\Phi, \Psi \in((\mathcal{N}) \otimes \mathcal{E})^{*}$ and $z \in \mathbb{R}$ if the series converges.

By similar arguments to those used for Theorem 6.3, we obtain the following theorem.

THEOREM 6.4. Suppose that for any $\xi \in \mathcal{N}$ and $p \geqslant 0$, there exist constants $C, K, q \geqslant 0$ such that

$$
\left|\frac{1}{n !}\left(M_{S \sigma(\xi)}^{r}\right)^{n} x\right|_{-q} \leqslant C e^{K|\xi|_{q}^{2}}|x|_{-p}, \quad x \in \mathcal{E}^{*} .
$$

Then for given $\Phi_{0} \in(\mathcal{N})^{*} \otimes \mathcal{E}^{*}$ and a continuous process $\{\Psi(t)\}_{t \in \mathbb{R}} \subset(\mathcal{N})^{*} \otimes \mathcal{E}^{*}$, the equation

$$
\frac{d \Phi(t)}{d t}=\Phi(t) \diamond_{\mathfrak{B}} \sigma+\Psi(t), \quad \Phi(0)=\Phi_{0},
$$

has a unique continuously differentiable solution $\Phi(t)$ in $(\mathcal{N})^{*} \otimes \mathcal{E}^{*}$ given by

$$
\begin{aligned}
\Phi(t)= & \Phi_{0} \diamond_{\mathfrak{B}} \overrightarrow{\operatorname{Wexp}}_{\mathfrak{B}}(t \sigma) \\
& +\left(\int_{0}^{t} \Psi(s) \diamond_{\mathfrak{B}} \overrightarrow{\operatorname{Wexp}}_{\mathfrak{B}}(-s \sigma) d s\right) \diamond_{\mathfrak{B}}{\overrightarrow{\operatorname{Wexp}_{\mathfrak{B}}}}(t \sigma)
\end{aligned}
$$

for all $t \in \mathbb{R}$.

EXAMPLE 6.1. As in Example 5.1, consider the Gel'fand triple

$$
(\mathcal{N}) \otimes \mathfrak{h} \subset \Gamma(H) \otimes \mathfrak{h} \subset(\mathcal{N})^{*} \otimes \mathfrak{h},
$$

where $\mathfrak{h}=M_{d \times d}(\mathbb{C})$. Let $\sigma \in(\mathcal{N})^{*} \otimes \mathfrak{h}$. If for any $\xi \in \mathcal{N}$ and $p \geqslant 0$, there exist constants $C, K, q \geqslant 0$ such that

$$
\frac{1}{n !}\|S \sigma(\xi)\|_{\mathrm{op}}^{n} \leqslant C e^{K|\xi|_{q}^{2}} \quad \text { for all } n \in \mathbb{N}
$$

then the unique solutions $\Phi(t)$ of $(6.1)$ and $(6.7)$ are given by $(6.6)$ and $(6.8)$, respectively. 
REMARK 6.1. All the results for the noncommutative case, i.e., when the continuous bilinear mapping $\mathfrak{B}$ is nonsymmetric, can be applied for the commutative case. Suppose the $\mathfrak{B}$-Wick product is commutative, i.e., $\mathfrak{B}$ is symmetric. Let $\{\sigma(t)\}_{t \in \mathbb{R}} \subset(\mathcal{N})^{*} \otimes \mathcal{E}^{*}$ be a continuous process. Suppose that for each $0 \leqslant t \leqslant T$ (for some $T>0$ ), any $\xi \in \mathcal{N}$ and any $p \geqslant 0$, there exist constants $C, K, q \geqslant 0$ such that

$$
\left|\frac{1}{n !}\left(M_{u}\right)^{n} x\right|_{-q} \leqslant C e^{K|\xi|_{q}^{2}}|x|_{-p}, \quad x \in \mathcal{E}^{*},
$$

where $u=S\left(\int_{0}^{t} \sigma(s) d s\right)(\xi)$ and $M_{u}:=M_{u}^{l}=M_{u}^{r}$. Then for each $\Phi_{0} \in$ $(\mathcal{N})^{*} \otimes \mathcal{E}^{*}$, the unique solution of the equation

$$
\frac{d \Phi(t)}{d t}=\sigma(t) \diamond_{\mathfrak{B}} \Phi(t)+\Psi(t), \quad 0 \leqslant t \leqslant T, \quad \Phi(0)=\Phi_{0},
$$

where $\{\Psi(t)\}_{0 \leqslant t \leqslant T} \subset(\mathcal{N})^{*} \otimes \mathcal{E}^{*}$ is continuous, is given by

$$
\Phi(t)=\Phi_{0} \diamond_{\mathfrak{B}} \exp ^{\diamond_{\mathfrak{B}}}\left(\int_{0}^{t} \sigma(s) d s\right)+\int_{0}^{t} \exp ^{\diamond_{\mathfrak{B}}}\left(\int_{s}^{t} \sigma(r) d r\right) \diamond_{\mathfrak{B}} \Psi(s) d s
$$

for all $t \in \mathbb{R}$, where $\exp ^{\diamond_{\mathfrak{B}}} \Psi:=\sum_{n=0}^{\infty} \frac{t^{n}}{n !} \Psi^{\diamond_{\mathfrak{B}} n}$.

\section{REFERENCES}

[1] K. Aase, B. Øksendal, N. Privault and J. Ubøe, White noise generalizations of the ClarkHaussmann-Ocone theorem with application to mathematical finance, Finance Stoch. 4 (2000), 465-496.

[2] L. Accardi, U. C. Ji and K. Saitô, Analytic characterizations of infinite dimensional distributions, Infin. Dimens. Anal. Quantum Probab. Related Topics 20 (2017), art. 1750007, 13 pp.

[3] F. E. Benth, T. Deck and J. Potthoff, A white noise approach to a class of non-linear stochastic heat equations, J. Funct. Anal. 146 (1997), 382-415.

[4] M. Bożejko, B. Kümmerer and R. Speicher, q-Gaussian processes: Non-commutative and classical aspects, Comm. Math. Phys. 185 (1997), 129-154.

[5] D. M. Chung and T. S. Chung, Wick derivations on white noise functionals, J. Korean Math. Soc. 33 (1996), 993-1008.

[6] D. M. Chung, U. C. Ji and N. Obata, Quantum stochastic analysis via white noise operators in weighted Fock space, Rev. Math. Phys. 14 (2002), 241-272.

[7] E. Effros and M. Popa, Feynman diagrams and Wick products associated with q-Fock space, Proc. Nat. Acad. Sci. USA 100 (2003), 8629-8633.

[8] M. Grothaus, Yu. G. Kondratiev and L. Streit, Regular generalized functions in Gaussian analysis. Infin. Dimens. Anal. Quantum Probab. Related Topics 2 (1999), 1-25.

[9] M. Grothaus, Yu. G. Kondratiev and G. F. Us, Wick calculus for regular generalized stochastic functionals, Random Oper. Stochastic Equations 7 (1999), 263-290.

[10] M. Grothaus and L. Streit, On regular generalized functions in white noise analysis and their applications, Methods Funct. Anal. Topology 6 (2000), 14-27.

[11] T. Hida, Analysis of Brownian Functionals, Carleton Math. Lect. Notes 13, Carleton Univ., Ottawa, 1975. 
[12] T. Hida, Brownian Motion, Springer, 1980.

[13] T. Hida, H.-H. Kuo, J. Potthoff and L. Streit, White Noise: An Infinite Dimensional Calculus, Kluwer, 1993.

[14] H. Holden, B. Øksendal, J. Ubøe, and T. Zhang, Stochastic Partial Differential Equations: A Modeling, White Noise Functional Approach, Birkhäuser, Boston, MA, 1996.

[15] R. L. Hudson and K. R. Parthasarathy, Quantum Ito's formula and stochastic evolutions, Comm. Math. Phys. 93 (1984), 301-323.

[16] U. C. Ji, Stochastic integral representation theorem for quantum semimartingales, J. Funct. Anal. 201 (2003), 1-29.

[17] U. C. Ji and E. Lytvynov, Wick calculus for noncommutative white noise corresponding to q-deformed commutation relations, Complex Anal. Oper. Theory 12 (2018), 1497-1517.

[18] U. C. Ji and N. Obata, A unified characterization theorem in white noise theory, Infin. Dimens. Anal. Quantum Probab. Related Topics 6 (2003), 167-178.

[19] U. C. Ji and N. Obata, Generalized white noise operators fields and quantum white noise derivatives, in: Analyse et Probabilités, Sém. Congr. 16, Soc. Math. France, 2007, 17-33.

[20] U. C. Ji and N. Obata, Annihilation-derivative, creation-derivative and representation of quantum martingales, Comm. Math. Phys. 286 (2009), 751-775.

[21] U. C. Ji and N. Obata, Implementation problem for the canonical commutation relation in terms of quantum white noise derivatives, J. Math. Phys. 51 (2010), art. 123507, 15 pp.

[22] U. C. Ji and N. Obata, Quantum white noise calculus and applications, in: Real and Stochastic Analysis, World Sci., Hackensack, NJ, 2014, 269-353.

[23] U. C. Ji and N. Obata, An implementation problem for boson fields and quantum Girsanov transform, J. Math. Phys. 57 (2016), art. 083502, 21 pp.

[24] U. C. Ji, N. Obata and H. Ouerdiane, Analytic characterization of generalized Fock space operators as two-variable entire functions with growth condition, Infin. Dimens. Anal. Quantum Probab. Related Topics 5 (2002), 395-407.

[25] I. Królak, Wick product for commutation relations connected with Yang-Baxter operators and new constructions of factors, Comm. Math. Phys. 210 (2000), 685-701.

[26] I. Kubo and S. Takenaka, Calculus on Gaussian white noise I-IV, Proc. Japan Acad. 56A (1980), 376-380; 411-416; 57A (1981), 433-437; 58A (1982), 186-189.

[27] H. -H. Kuo, White Noise Distribution Theory, CRC Press, 1996.

[28] N. A. Kachanovsky, An extended stochastic integral and a Wick calculus on parametrized Kondratiev-type spaces of Meixner white noise, Infin. Dimens. Anal. Quantum Probab. Related Topics 11 (2008), 541-564.

[29] Y. G. Kondratiev, P. Leukert and L. Streit, Wick calculus in Gaussian analysis, Acta Appl. Math. 44 (1996), 269-294.

[30] P.-A. Meyer, Quantum Probability for Probabilists, Lecture Notes in Math. 1538, Springer, 1993.

[31] N. Obata, Operator calculus on vector-valued white noise functionals, J. Funct. Anal. 121 (1994), 185-232.

[32] N. Obata, White Noise Calculus and Fock Space, Lecture Notes in Math. 1577, Springer, 1994.

[33] N. Obata, Generalized quantum stochastic processes on Fock space, Publ. RIMS Kyoto Univ. 31 (1995), 667-702.

[34] N. Obata, Constructing one-parameter transformations on white noise functions in terms of equicontinuous generators, Monatsh. Math. 124 (1997), 317-335.

[35] N. Obata, Wick product of white noise operators and quantum stochastic differential equations, J. Math. Soc. Japan 51 (1999), 613-641.

[36] N. Obata and H. Ouerdiane, A note on convolution operators in white noise calculus, Infin. Dimens. Anal. Quantum Probab. Related Topics 14 (2011), 661-674.

[37] K. R. Parthasarathy, An Introduction to Quantum Stochastic Calculus, Birkhäuser, 1992.

[38] J. Potthoff and L. Streit, A characterization of Hida distributions, J. Funct. Anal. 101 (1991), 212-229. 
[39] J. Potthoff and M. Timpel, On a dual pair of spaces of smooth and generalized random variables, Potential Anal. 4 (1995), 637-654.

Un Cig Ji

Chungbuk National University

Department of Mathematics

Institute for Industrial and Applied Mathematics

Chungbuk National University

Cheongju 28644, Korea

E-mail: uncigji@chungbuk.ac.kr
Peng Cheng Ma Chungbuk National University Department of Mathematics Chungbuk National University

Cheongju 28644, Korea E-mail: mapengcheng@ chungbuk.ac.kr

Received 5.8.2020;

revised version 12.11.2020 